\title{
Pion form factor from RBC and UKQCD
}

\author{
Andreas Jüttner* \\ Institut für Kernphysik, Johannes Gutenberg Universität, \\ Johann-Joachim-Becher Weg 45, 55116 Mainz, Germany, \\ E-mail: juettner@kph.uni-mainz.de
}

\section{P.A. Boyle, C. Kelly, C. Maynard, J.M. Zanotti}

School of Physics, The University of Edinburgh,

Edinburgh, EH9 3JZ, UK

\section{J.M. Flynn, H.P. de Lima, C.T. Sachrajda \\ School of Physics and Astronomy, University of Southampton, \\ Southampton, SO17 1BJ, UK,}

\begin{abstract}
We present computations of the electromagnetic pion form factor for various pion masses and at low values of $Q^{2} \equiv-q^{2}$, where $q$ is the momentum transfer. We used RBC+UKQCD's gauge configurations of Domain Wall fermions. In order to be able to reach low momentum transfers we employ partially twisted boundary conditions using the techniques we had developed and tested earlier.
\end{abstract}

6th International Workshop on Chiral Dynamics, CD09

July 6-10, 2009

Bern, Switzerland

${ }^{*}$ Speaker. 


\section{Introduction}

With the constant improvement of phenomenological applications of lattice QCD also the computation of the pion vector form factor and in particular the pion's charge radius is now available from a number of groups $[1,2,3,4,5,6]$ with unprecedented quality. Dynamical sea quarks ( $N_{f}=2$ and $\left.N_{f}=2+1\right)$ are standard by now and results are available for a range of pion masses down to about $250 \mathrm{MeV}$. Systematic errors like cut-off effects and finite volume effects are under good control. In this talk we presented the computation of the two quantities by the RBC+UKQCD collaboration. The special feature of this computation is that by using suitable boundary conditions for the finite volume valence quark fields $[7,8,9,10,11,12,13,14,15]$ it is possible to compute the pion form factor at arbitrarily small momentum transfers. This provides for the first time a fine grained momentum resolution for $Q^{2} \rightarrow 0$ and hence a clean and parametrization-independent definition of the pion's charge radius. We investigate the quality of various ansaetze for the $Q^{2}$ dependence of the form factor and we use NLO chiral perturbation theory to extrapolate the charge radius at one of our simulation points, $m_{\pi}=330 \mathrm{MeV}$, to the physical pion mass.

\section{Computational strategy}

The pion form factor $f^{\pi \pi}\left(q^{2}\right)$ defined through the QCD transition matrix element

$$
\left\langle\pi\left(p^{\prime}\right)\left|V_{\mu}\right| \pi(p)\right\rangle=f^{\pi \pi}\left(q^{2}\right)\left(q^{2}\right)\left(p+p^{\prime}\right)_{\mu},
$$

where $V_{\mu}$ is the electromagnetic current, can be extracted from suitable Euclidean 2pt- and 3ptcorrelation functions computed on the lattice (cf. [4]); the pions charge radius is defined as

$$
\left\langle r_{\pi}^{2}\right\rangle_{V}=\left.6 \frac{d}{d q^{2}} f^{\pi \pi}\left(q^{2}\right)\right|_{q^{2}=0} .
$$

In a finite volume of spatial extent $L$ with periodic boundary conditions for the quark fields the allowed quark 3-momenta are $\vec{p}_{\vec{n}}=\vec{n} 2 \pi / L$, where $\vec{n}$ is a vector of integers and correspondingly the finite volume pion energies are $E_{\pi}\left(\vec{p}_{\vec{n}}\right)=\sqrt{m_{\pi}^{2}+\left(\vec{p}_{\vec{n}}\right)^{2}}$. Given the dependence of the momentum transfer $q=-Q$ in (2.1) on the pion's momenta,

$$
q^{2}=\left(p-p^{\prime}\right)^{2}=\left(E_{\pi}\left(\vec{p}_{\vec{n}}\right)-E_{\pi}\left(\vec{p}_{\vec{n}}^{\prime}\right)\right)^{2}-\left(\vec{p}_{\vec{n}}-\vec{p}_{\vec{n}^{\prime}}^{\prime}\right)^{2},
$$

one finds, that the lowest accesible value is $Q_{\min }^{2}=-2 m_{\pi}\left(m_{\pi}-\right.$ $\sqrt{m_{\pi}^{2}+(2 \pi / L)^{2}}$. Naively, for typical simulation parameters like the ones used for the study presented here, $Q_{\min }^{2} \approx 0.15 \mathrm{GeV}^{2}$ which is in a range where chiral perturbation theory may cease to provide a reliable description of QCD.

In order to compute $f^{\pi \pi}\left(q^{2}\right)$ also in the range $0<Q^{2}<Q_{\min }^{2}$ we use partially twisted boundary conditions [9], combining gauge field configurations generated with sea quarks obeying periodic boundary conditions with valence quarks with twisted boundary conditions $[7,8,9,10,11,12,13$, 14 , 15], i.e. the valence quarks satisfy $q\left(x_{k}+L\right)=e^{i \theta_{k}} q\left(x_{k}\right),(k=1,2,3)$, where $\theta_{k}$ is the twisting angle in the $\hat{k}$-direction. We have demonstrated in section 2.3 of ref. [4] that it is possible to introduce twisted boundary conditions independently for the three valence quarks and antiquarks, i.e. $\vec{\theta}_{1}$ for $q_{1}, \vec{\theta}_{2}$ for $q_{2}$ and $\vec{\theta}_{3}$ for $q_{3}$ in figure 1 . In our study it will be sufficient to set $\vec{\theta}_{3}=0$ so that 


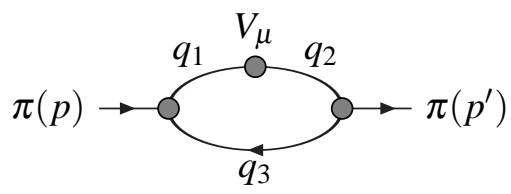

Figure 1: Sketch of the valence quark flow in the electromagnetic form factor of the pion. There is a similar contribution in which the current is on the antiquark line and the spectator is a quark.

\begin{tabular}{lcc}
\hline \hline & $\mathrm{A}$ & $\mathrm{B}$ \\
\hline$a / \mathrm{fm}$ & $\approx 0.1$ & $\approx 0.1$ \\
$(L / a)^{3} \times T / a \times L_{s}$ & $16^{3} \times 32 \times 8$ & $24^{3} \times 64 \times 16$ \\
$a m_{s}^{\text {sea }}$ & 0.04 & 0.04 \\
$a m_{q}^{\text {sea }}$ & $0.02 / 0.01$ & 0.005 \\
$m_{\pi} / \mathrm{MeV}$ & $590 / 480$ & 330 \\
\hline \hline
\end{tabular}

Table 1: Parameters for the two studies with $N_{f}=2+1$ Domain Wall fermions.

the spectator quark or antiquark satisfies periodic boundary conditions. By varying $\vec{\theta}_{1}$ and $\vec{\theta}_{2}$ we are able to tune the momentum of the charged pion continuously. With this technique the dispersion relation for a meson with twisting angle $\vec{\theta}$ takes the form [8, 12], $E_{\pi}(\vec{p})=\sqrt{m_{\pi}^{2}+\left(\vec{p}_{\vec{n}}+\frac{\vec{\theta}}{L}\right)^{2}}$. For the matrix element in (2.1) with the initial and the final meson carrying momenta $\vec{p}=\vec{p}_{\vec{n}}+\vec{\theta} / L$ and $\vec{p}^{\prime}=\vec{p}_{\vec{n}^{\prime}}+\vec{\theta}^{\prime} / L$ respectively, the momentum transfer between the initial and the final state meson is

$$
q^{2}=\left(p-p^{\prime}\right)^{2}=\left(E_{\pi}\left(\vec{p}_{\vec{n}}\right)-E_{\pi}\left(\vec{p}_{\vec{n}}^{\prime}\right)\right)^{2}-\left(\left(\vec{p}_{\vec{n}}+\vec{\theta} / L\right)-\left(\vec{p}_{\vec{n}^{\prime}}^{\prime}+\vec{\theta}^{\prime} / L\right)\right)^{2} .
$$

The finite-volume corrections with partially twisted boundary conditions decrease exponentially with $L$ similarly to those with periodic boundary conditions $[9,16]$.

\section{Results}

We carried out two simulations: Study A was exploratory with relatively heavy pions and in small volume. Study B was done with a lighter pion mass and in a larger volume where finite volume effects are expected to be small. We summarize the simulation parameters in table 1 . The result of study A is illustrated in the plots in figure 2: The left hand side plot shows the pion form factor for the two simulated pion masses together with the results for pole-fits:

$$
f^{\pi \pi}\left(q^{2}\right)=\frac{1}{1-q^{2} / M_{\text {pole }}^{2}}
$$

The vertical lines correspond to the values of $Q_{\min }^{2}$ for the two sea quark masses, respectively. The data points left of the vertical line could only be obtained using partially twisted boundary conditions. The right hand side plot shows the results for the charge radius (same color coding) as 

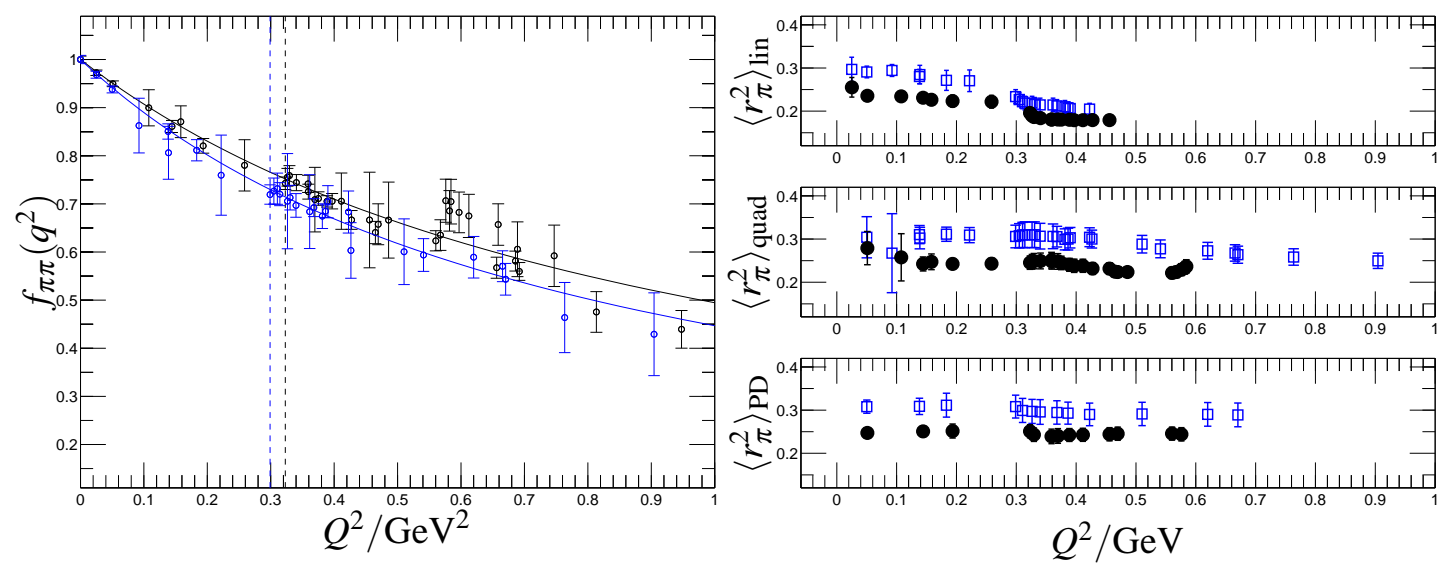

Figure 2: Results of the exploratory study A. L.h.s. plot: Results for $a m_{l}=0.02$ and $a m_{l}=0.01$ (black, blue), respectively; r.h.s. plot: results for the charge radious for various fit-ansätze and various fit ranges.
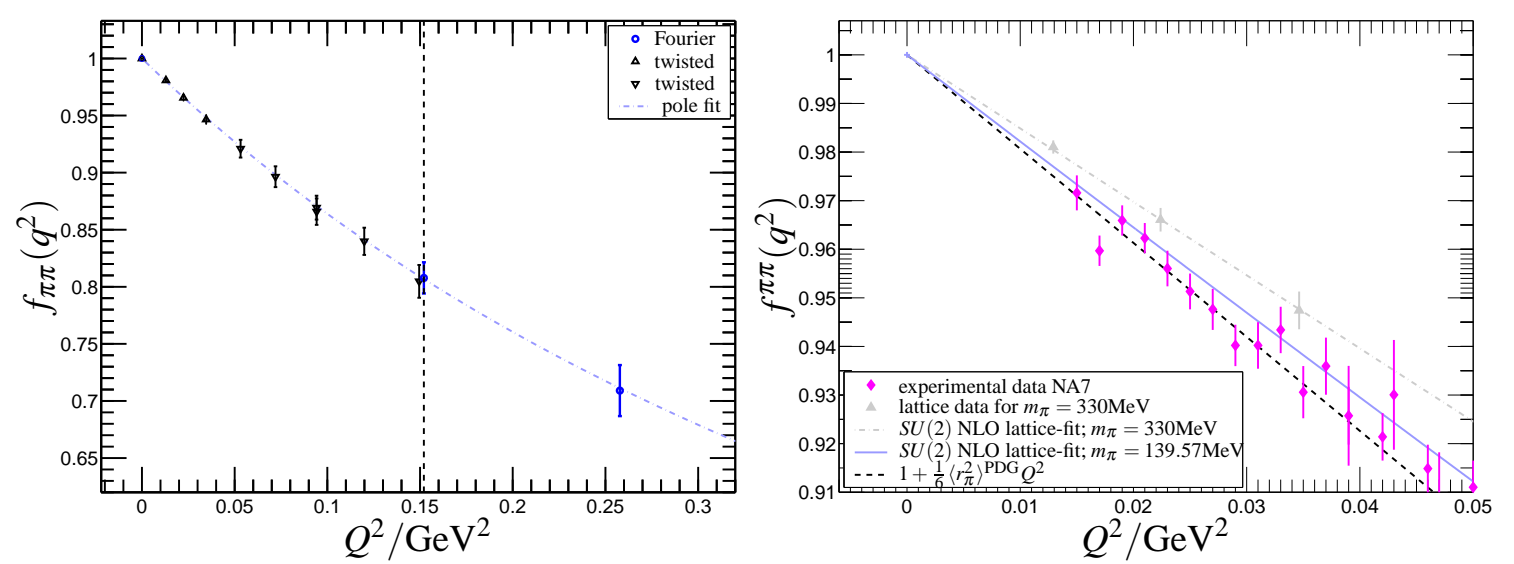

Figure 3: Study B: L.h.s.: Form factor as a function of the momentum transfer. R.h.s.: Comparison of experimental results (magenta diamonds) for the form factor $f^{\pi \pi}\left(q^{2}\right)$, lattice results at $m_{\pi}=330 \mathrm{MeV}$ (grey triangles and dash-dotted grey line) and the extrapolation of the lattice results to the physical point (blue solid line) using NLO $S U(2)$ chiral perturbation theory. In addition we also represent the PDG world average for the charg radious in terms of the black dashed line.

obtained for various fit ansätze: linear in $q^{2}$, 2nd order polynomial in $q^{2}$, pole dominance fit like in eqn. (3.1). The values on the $x$-axis indicate up to which value of $Q^{2}$ data points have been included into the fit. All three fits turn out to be rather stable for a range of small momentum transfers; the stability of the pole dominance fit extends over the largest range of momentum transfers. The plots in figure 3 show the results of study B. On the 1.h.s., again the vertical line indicates the value of $Q_{\min }^{2}$. The blue data points (circles) have been obtained by Fourier transformation and all black points (triangles) have been obtained using partially twisted boundary conditions. Note the agreement between the two results at $Q_{\max }^{2}$ which have been obtained with the two different ways of inducing momenta.

The presented data for the form factor and the charge radius is for unphysically heavy pions. Since we think that the pion masses in study A are too large to be described correctly by chiral perturbation theory we here present our attempt to extrapolate the results for the charge radius in 


\begin{tabular}{lllll}
\hline \hline collaboration & technique & $\left\langle r_{\pi}^{2}\right\rangle_{\chi}\left[\mathrm{fm}^{2}\right]$ \\
\hline PDG & & & $0.452(11)$ \\
\hline QCDSF/UKQCD & {$[2]$} & $N_{f}=2$ & Clover & $0.441(19)$ \\
JLQCD & {$[1]$} & $N_{f}=2$ & Clover & $0.396(10)$ \\
JLQCD & {$[3]$} & $N_{f}=2$ & Overlap & $0.409(44)$ \\
ETM & {$[6]$} & $N_{f}=2$ & twisted mass & $0.456(38)$ \\
RBC+UKQCD & {$[5]$} & $N_{f}=2+1$ & Domain Wall & $0.418(31)$ \\
\hline \hline
\end{tabular}

Table 2: Summary of current lattice results for the pion's charge radius in comparison to the PDG-average.

study B to the physical point using NLO SU(2) chiral perturbation theory, [17],

$$
\left\langle r_{\pi}^{2}\right\rangle_{\mathrm{SU}(2), \mathrm{NLO}}=-\frac{12 l_{6}^{r}}{f^{2}}-\frac{1}{8 \pi^{2} f^{2}}\left(\log \frac{m_{\pi}^{2}}{\mu^{2}}+1\right),
$$

where $f$ is the decay constant in the chiral limit, $l_{6}^{r}$ is the only other low energy constant at this order and $\mu$ is the renormalization scale. We note that the results of study $\mathrm{B}$ alone do not allow to assess whether the mass dependence of the lattice data is properly described by NLO chiral perturbation theory. We also carried out a fit using SU(3) chiral perturbation theory [18] and results can be found in [5]. Based on the experience of ref. [19], for our best estimate we take the result from the fit to the $\mathrm{SU}(2)$ expression at NLO including the three data points at $Q^{2}=0.013,0.022$ and $0.035 \mathrm{GeV}$,

$$
\left.\left\langle r_{\pi}^{2}\right\rangle\right|_{m_{\pi}=330 \mathrm{MeV}}=0.354(31) \mathrm{fm}^{2} \rightarrow l_{6}^{r}\left(m_{\rho}\right)=-0.0093(9),\left\langle r_{\pi}^{2}\right\rangle=0.418(31) \mathrm{fm}^{2} .
$$

These results are summarized in the r.h.s. plot in figure 3: In addition to our lattice results and NLO ChiPT fit in grey, the result for the chiral extrapolation (light blue solid line) is compared to the experimental data (magenta) [20] and the PDG's pole dominance fit. Our chirally extrapolated form factor drops less rapidly with $Q^{2}$ than the PDG-fit but as the plot on the r.h.s. of table 2 shows, our resulting value for the charge radius is compatible with the PDG-average. The same plot shows other lattice results and the PDG-average.

For study B we used propagators generated from a single time-slice stochastic source. We compared the cost, obtaining similar errors for the pion mass, the normalization constant of the vector current, $Z_{V}$, and the pion's electromagnetic form factor at $Q_{\min }^{2}$, finding a gain of approximately a factor of 12 in favour of the noise source propagators. A gain was also found in the study presented by the ETMC collaboration in [6] and in the recent publication by the UKQCD collaboration [21].

\section{Summary and Outlook}

In this paper we have successfully used partially twisted boundary conditions to compute the electromagnetic form factor of a pion for $Q^{2} \rightarrow 0$. We use our results to compute the low energy constant $l_{6}^{r}$ of NLO SU(2) and then to determine the physical form factor and charge radius. The results which we obtain are in good agreement with the experimentally determined form factor 
which gives us further confidence in the use of chiral perturbation theory in the mass range below $330 \mathrm{MeV}$.

We are currently analyzing a set of configurations with the same action on a $32^{3}$ lattice with a finer lattice spacing and will repeat the present calculation with this ensemble. Although the mass and momentum transfers are sufficiently small to expect that NLO SU(2) ChPT is a good approximation, it would be nice to be able to check this explicitly by computing $f^{\pi \pi}\left(q^{2}\right)$ for a range of pion masses. We are planning to carry out such an analysis in the future.

\section{Acknowledgments}

We warmly thank Dirk Brömmel for informative discussions about the content of [2] and Bálint Joó for help in learning to use Cray XT4 systems. We are very grateful to the Engineering and Physical Sciences Research Council (EPSRC) for a substantial allocation of time on HECToR under the Early User initiative. We thank Arthur Trew, Stephen Booth and other EPCC HECToR staff for assistance and EPCC for computer time and assistance on BlueGene/L.

The calculations also made use of QCDOC computers, and we thank the support staff in the $\mathrm{ACF}$ at Edinburgh and at BNL. The QCDOC development and the resulting computer equipment used in this calculation were funded by the U.S. DOE grant DE-FG02-92ER40699, PPARC JIF grant PPA/J/S/1998/00756 and by RIKEN. This work was supported by PPARC grants PPA/G/O/ 2002/00465, PP/D000238/1 and PP/C504386/1. Our calculations made use of the CHROMA[22] and BAGEL software packages.

JMF, AJ, HPdL and CTS acknowledge support from STFC Grant PP/D000211/1 and from EU contract MRTN-CT-2006-035482 (Flavianet). PAB, CK, CMM and JMZ acknowledge support from STFC grant PP/D000238/1.

\section{References}

[1] JLQCD, S. Hashimoto et al., Pion form factors in two-flavor QCD, PoS LAT2005 (2006) 336, hep-lat/0510085.

[2] QCDSF/UKQCD, D. Brommel et al., The pion form factor from lattice QCD with two dynamical flavours, Eur. Phys. J. C51 (2007) 335, hep-lat/0608021.

[3] JLQCD, S. Aoki et al., Pion form factors from two-flavor lattice QCD with exact chiral symmetry, (2009), 0905.2465.

[4] P.A. Boyle et al., Hadronic form factors in lattice QCD at small and vanishing momentum transfer, JHEP 05 (2007) 016, hep-lat/0703005.

[5] P.A. Boyle et al., The pion's electromagnetic form factor at small momentum transfer in full lattice QCD, JHEP 07 (2008) 112, 0804.3971.

[6] R. Frezzotti, V. Lubicz and S. Simula, Electromagnetic form factor of the pion from twisted-mass lattice QCD at Nf=2, (2008), 0812.4042.

[7] P.F. Bedaque, Aharonov-Bohm effect and nucleon nucleon phase shifts on the lattice, Phys. Lett. B593 (2004) 82, nucl-th/0402051.

[8] G.M. de Divitiis, R. Petronzio and N. Tantalo, On the discretization of physical momenta in lattice QCD, Phys. Lett. B595 (2004) 408, hep-lat/0405002. 
[9] C.T. Sachrajda and G. Villadoro, Twisted boundary conditions in lattice simulations, Phys. Lett. B609 (2005) 73, hep-lat/0411033.

[10] P.F. Bedaque and J.W. Chen, Twisted valence quarks and hadron interactions on the lattice, Phys. Lett. B616 (2005) 208, hep-lat/0412023.

[11] B.C. Tiburzi, Twisted quarks and the nucleon axial current, Phys. Lett. B617 (2005) 40, hep-lat/0504002.

[12] UKQCD, J.M. Flynn, A. Jüttner and C.T. Sachrajda, A numerical study of partially twisted boundary conditions, Phys. Lett. B632 (2006) 313, hep-lat/0506016.

[13] D. Guadagnoli, F. Mescia and S. Simula, Lattice study of semileptonic form factors with twisted boundary conditions, Phys. Rev. D73 (2006) 114504, hep-lat/0512020.

[14] B.C. Tiburzi, Flavor twisted boundary conditions and isovector form factors, Phys. Lett. B641 (2006) 342, hep-lat/0607019.

[15] T.B. Bunton, F.J. Jiang and B.C. Tiburzi, Extrapolations of lattice meson form factors, Phys. Rev. D74 (2006) 034514, hep-lat/0607001.

[16] F.J. Jiang and B.C. Tiburzi, Flavor Twisted Boundary Conditions, Pion Momentum, and the Pion Electromagnetic Form Factor, Phys. Lett. B645 (2007) 314, hep-lat/0610103.

[17] J. Gasser and H. Leutwyler, Chiral Perturbation Theory to One Loop, Ann. Phys. 158 (1984) 142.

[18] J. Gasser and H. Leutwyler, Chiral Perturbation Theory: Expansions in the Mass of the Strange Quark, Nucl. Phys. B250 (1985) 465.

[19] RBC-UKQCD, C. Allton et al., Physical Results from 2+1 Flavor Domain Wall QCD and SU(2) Chiral Perturbation Theory, Phys. Rev. D78 (2008) 114509, 0804.0473.

[20] NA7, S.R. Amendolia et al., A measurement of the space - like pion electromagnetic form-factor, Nucl. Phys. B277 (1986) 168.

[21] P.A. Boyle et al., Use of stochastic sources for the lattice determination of light quark physics, JHEP 08 (2008) 086, 0804.1501.

[22] SciDAC, R.G. Edwards and B. Joo, The Chroma software system for lattice QCD, Nucl. Phys. Proc. Suppl. 140 (2005) 832, hep-lat/0409003. 